\title{
¿Método tradicional o alternativo? Hacia la consolidación de una estrategia didáctica para el aprendizaje del tema segunda guerra mundial acorde a las necesidades educativas actuales
}

\section{Traditional or alternative method? Towards the consolidation of a didactic strategy for learning the theme of the Second World War according to current educational needs}

Wilmer Edilson Cuellar Sambony

Corporación Universitaria Minuto de Dios, Colombia

Autor por correspondencia: wilmere_901@hotmail.com

Fecha de recepción: 11 de septiembre de 2018 - Fecha de aceptación: 01 de diciembre de 2018

Resumen: La Segunda Guerra Mundial, un acontecimiento de discusiones políticas y dominio social que parte la historia en dos y finaliza con la creación de nuevas instituciones que establecen un orden mundial. En el ámbito educativo actualmente predomina el método de enseñanza tradicional el cual fue pensado para responder en las necesidades de un contexto, por ello es pertinente y necesario se dé un proceso de transición en los métodos. Para alcanzar el objetivo propuesto, se desarrollan los referentes teóricos y se selecciona la metodología cuantitativa de tipo experimental con la cual se usan como instrumentos para la recolección de información el cuestionario, la observación y una guía didáctica la cual integra varias estrategias didácticas. Cabe mencionar que como muestra se selecciona los dos grados octavos del colegio Cooperativo la Presentación integrado por 43 estudiantes, un grupo llamado control realiza su proceso de aprendizaje mediante el método tradicional y el otro grupo experimental mediante las estrategias didácticas de las TIC, la metodología por proyectos y el trabajo colaborativo-cooperativo. Luego de desarrollar las actividades por medio de las estrategias didácticas y recolectar la información encontramos que el grupo experimental tiene motivación, autonomía, interés, inquietud por desarrollar su proceso de aprendizaje cuando se emplea esta metodología a diferencia del grupo control que, aunque con resultados académicos buenos los factores mencionados no son evidentes durante el proceso de enseñanza.

Palabras claves: educación; tic; metodología por proyectos; trabajo colaborativo; cooperativo; estrategias didácticas; segunda guerra mundial

Abstract: The Second World War, an event of political discussions and social domination that split the story in two and ends with the creation of new institutions that establish a world order. In the educational field, the traditional teaching method is currently predominant, which was thought to respond to the needs of a context, so it is pertinent and necessary to have a process of transition in the methods. To reach the proposed objective, theoretical referents are developed and the quantitative methodology of experimental type is selected, with which the questionnaire, observation and a didactic guide which integrates several didactic strategies are used as instruments for collecting information. It is worth mentioning that as a sample the two eighth grades of the Cooperative School are selected, the Presentation is composed of 43 students, a group 
called control performs its learning process through the traditional method and the other experimental group through the didactic strategies of the ICT, the methodology by projects and cooperative-cooperative work. After developing the activities by means of the didactic strategies and collecting the information we found that the experimental group has motivation, autonomy, interest, restlessness to develop their learning process when this methodology is used, unlike the control group that, although with academic results Good the mentioned factors are not evident during the teaching process.

Key words: education; icts; project-based learning; collaborative; cooperative work; teaching strategies; second world war

\section{Introducción}

La Segunda Guerra Mundial, un acontecimiento de discusiones políticas y dominio social que parte la historia en dos y finaliza con la creación de nuevas instituciones que establecen un orden mundial, las cuales toman como referente hechos pasados como la primera guerra, la guerra fría y la segunda guerra mundial para crear políticas y planes de acción con el propósito de evitar sucesos como los vividos. Siendo así, que es importante conocer todo lo relacionado con la guerra en especial las consecuencias con el fin de hacer un análisis de la realidad y del contexto desde una época histórica y así valorar lo que hasta ahora se ha hecho, evitando llegar a caer en la provocación de desear una tercera guerra mundial, que vista de

Desde todas las ignorancias las consecuencias no se dimensionan, pero si vemos desde la historia, la política y sociedad no es conveniente para ningún Estado, estalle una tercera guerra mundial. (CIDEAD, 2005)

Fue seleccionado este grado debido a que el tema segunda Guerra Mundial se desarrolla según el plan de estudios en el grado octavo además porque mediante el conocimiento del tema se hace un análisis de la realidad, contexto de la época con el fin de desarrollar el pensamiento crítico, propositivo y habilidades para vivir en sociedad, por ello desde el conocimiento de la realidad se valora lo que hasta ahora se ha hecho en el mundo luego de este acontecimiento que es el de evitar otra guerra, la cual genera consecuencias que esta generación al no tener conocimiento de la historia no dimensionaría.

Partiendo de las investigaciones realizadas y de la praxis, el método que predomina en la enseñanza de las ciencias sociales es el tradicional; es de anotar que este método fue pensado desde un contexto el cual respondía a unas necesidades educativas específicas. No obstante, teniendo en cuenta la demanda educativa actual puede verse que el joven actual es distinto, por tanto, difícilmente se acopla al modelo de enseñanza tradicional, de allí que requiera estrategias de enseñanza distintas (Fernández Muñoz, 2010 \& Marc Prenskey, 2010).

Dentro de las estrategias didácticas empleadas en el sistema educativo tradicional vigente se encuentra la clase magistral, la exposición, el desarrollo de talleres, realización de trabajos externos, lecturas, resúmenes, esquemas, mapas conceptuales en los cuales se debe centrar el interés y atención para lograr el desarrollo del pensamiento crítico-social.

Las dificultades presentadas en el proceso de aprendizaje de las ciencias sociales en el grado octavo tienen su origen en el desinterés debido a que conciben el área como teórica, de 
difícil comprensión, de poco material didáctico de apoyo, poca motivación por parte del docente para aprender, prejuicio, método de enseñanza tradicional el cual no logra motivar y generar aprendizajes significativos a la hora de aprender historia siendo esta la causa de los bajos resultados en las pruebas de estado a nivel institucional.

Se desarrolla la presente investigación porque es necesaria una transición desde el método tradicional ejemplo de ello son los materiales educativos como los textos físicos que están siendo remplazados por el hipertexto/hipermedia los cuales han modificado la experiencia de leer, escribir y aprender (Landow, 1995). De allí que sea importante y oportuno utilizar las herramientas que con el avance tecnológico se ponen a disposición de la academia.

\section{Marco teórico}

Las TIC se han convertido en un desafío para la educación, debido a que estamos expuestos a estímulos como la televisión, el internet, el teléfono celular, el computador, la consola de videojuegos los cuales tienen repercusiones sobre el proceso formativo (Monsalve Ochoa, 2011). De acuerdo con Morrissey (2010) el uso de las TIC trae ventajas como: la motivación para los estudiantes ya que brinda aprendizajes activos, permiten trabajar los contenidos con diferentes estrategias, pueden realizarse los procesos de evaluación, se atienden dificultades de aprendizaje asociadas a la inclusión social e igualdad de oportunidades, apoya el trabajo individual y grupal, ofrece simulaciones, mapas, animaciones, se crean situaciones que desafían el conocimiento, entre otros. Para Cabero Almenara (2014) el uso de las TIC en el aula de clase depende en gran medida de la capacidad que tiene el docente de utilizarlas.

El Aprendizaje Basado en Proyectos (ABP) tiene sus bases en el constructivismo en el que el conocimiento según Villar Sola (2013) citando a Gergen (2007) define el ABP como un conjunto de elementos teóricos que se basan en la construcción y la resolución de problemas de aula, con la intención que el estudiante reciba los conocimientos para que así los pueda trasladar fuera del aula; Por medio de este modelo el estudiante en grupos de trabajos es capaz de resolver, diseñar, evaluar problemas y proyectos que le permiten construir conocimiento, trabajar colaborativamente, desarrollar habilidades y estar comprometidos con el proceso de enseñanza aprendizaje (Villar Sola, 2013). Con la metodología por proyectos el estudiante pasa a ser el protagonista del proceso de enseñanza y aprendizaje y el profesor a ser mediador de la realidad con el ámbito académico. (Austin, Abella, Delgado, \& Hortiguela, 2016)

Este modelo de aprendizaje exige que el profesor sea creador, guía y a su vez estimule a los estudiantes para investigar, descubrir y construir, teniendo así, un acercamiento desde el ámbito académico a la realidad concreta, por medio del desarrollo de un trabajo (Maldonado Pérez, 2008).

Partiendo de los referentes teóricos del constructivismo en el que el aprendizaje se construye a partir de la relación significativa de la información, conocimientos previos y contexto y la premisa la escuela debe cambiar porque la sociedad cambia, las estrategias didácticas de las TIC, metodología por proyectos y trabajo colaborativo cooperativo han sido empleadas según queda evidenciado en la investigación de; Martinez, B Gomez (2014) Las Tic como herramienta para fortalecer el aprendizaje colaborativo, en las IES, en la cual se demuestra 
que mediante el empleo de las TIC se logra alcanzar mejores aprendizajes que con el método tradicional, Mur, L (2015) El aprendizaje de la historia con wiki en educación secundaria, en la cual se evidencia un aumento en la motivación de los estudiantes por aprender debido al cambio metodológico en el cual se empleó la wiki y se transformó el rol del profesor Murcia,Y; Tejedor, M.; Lancheros, D.(2017) Impacto de una herramienta multimedia en el proceso de enseñanza aprendizaje de la historia en el aula, muestra como el empleo de una aula dinámica la cual se compone de momentos como: motivación, síntesis, creatividad, evaluación, retroalimentación, mejora los resultados de aprendizaje, Moreno, J.; Vera, M.; Soriano, M.; Seva, F.; Quiñonero, F.; Ibañez, M. (2015) La enseñanza de la historia a través de las tecnologías, la creatividad y el trabajo colaborativo, muestran que mediante el empleo de programas como Photopeach, Glogster con los cuales los estudiantes se desarrollan rompecabezas, videos, folletos, comparaciones se logra desarrollar el pensamiento crítico y reflexivo en el área de ciencias sociales y Casadiego Cabrales, Alix Maria (2016) Efectividad del uso de herramientas web utilizando las estrategias de aprendizaje colaborativo, en el que se compara el aprendizaje tradicional y el mediado por tic, evidencia que el uso de la web incremental el nivel de conocimiento que para el caso se dio a través del uso de la wiki, foro, chat y correo los cuales facilitaron el aprendizaje para en los estudiantes con rendimiento académico bajo.

\section{Diseño metodológico}

La investigación se clasifico de tipo cuantitativa y método experimental ya que según Tamayo es el procedimiento más indicado para hallar relaciones de causa - efecto debido a que trabaja con el control de una o más variables a las cuales el investigador provoca alteraciones con el propósito de observar los cambios (Marín Gallego, 2012) y debido a que el objetivo general fue el de implementar las TIC, la metodología por proyectos, el trabajo cooperativo-colaborativo como estrategias didácticas para el aprendizaje del tema segunda guerra mundial, en este sentido se analizó la efectividad de dos métodos de enseñanza aprendizaje mediante el sistema tradicional y el mencionado.

La muestra objeto de estudio estuvo conformada por cuarenta y seis (46) estudiantes pertenecientes al grado octavo con edades comprendidas entre 13 y 15 años, los cuales se clasificaron en dos grupos de veintitrés (23) estudiantes cada uno. el control desarrollo la temática por el sistema tradicional (aula, docente, tablero y grupo) y el grupo experimental empleo una dinámica que gira en torno al empleo de las TIC, metodología por proyectos, el trabajo colaborativo-cooperativo.

Se aplicó a los grupos una prueba estructurada la cual constaba de 25 preguntas de selección múltiple de las cuales como criterio de calificación mínimo para aprobar se establecen 15 preguntas correctas, con el fin determinar los conocimientos previos, adquiridos y analizar la efectividad de las estrategias didácticas empleadas.

El grupo control desarrollo su proceso de aprendizaje del tema Segunda Guerra Mundial mediante el sistema tradicional atreves del uso del tablero, las exposiciones y la clase magistral, este proceso se realizó durante tres semanas en el aula de clase normal la cual se caracteriza por tener escritorios individuales colocados en filas, un tablero y tres ventiladores, con una intensidad horaria de 4 horas cada una. Las temáticas desarrolladas fueron antecedentes, inicio 
de la guerra, campos de concentración, Pearl Harbor- Midway, Batalla de Moscú, Bloqueo de Leningrado, Protagonistas, Batalla de Kurskaya, Guerra en el desierto, Stalingrado, Liberación de Europa, Toma de Berlín.

Con el grupo experimental se utilizó un guion de trabajo para el aprendizaje del trema Segunda Guerra Mundial en el cual se integran las estrategias didácticas de las TIC, el trabajo colaborativo - cooperativo, este se estructura en fases de trabajo Búsqueda, clasificación de la información, clasificación, lectura y síntesis de la información, estructura video, elaboración edición del video -publicación, socialización videos y aplicación pos test; en el cual se solicita como producto final la elaboración de un video sobre la Segunda Guerra Mundial en el que se integren los subtemas mencionados en el párrafo anterior. El guion es desarrollado con los computadores de la sala de sistemas del colegio la cual tiene aire acondicionado, treinta computadores con sistema operativo Windows XP, disco duro de $500 \mathrm{~Gb}$ de disco duro, $4 \mathrm{~Gb}$ de memoria RAM, Intel i5 de segunda generación, todos conectados a la red de internet de banda ancha de $5 \mathrm{MB}$ y los dispositivos móviles de los estudiantes como Tablet con Android 5.0, celulares gama media y alta entre ellos de las marcas Samsung, LG, huawei, IPhone, entre otros y portátiles de las marcas Lenovo, HP, Toshiba, Apple con sistema operativo Windows vista, Windows 10 y MacOs.

El trabajo se caracterizó porque en cada clase los estudiantes llegaban a la sala de sistemas y organizaban el plan de trabajo diario, ello lo hacía por medio del diálogo en el cual discutían sobre lo realizado, aclaraban inquietudes y organizaban las ideas de lo que realizarían en la clase. Dentro de las fuentes de información y consulta se encuentra educatina, Wikipedia, Slideshare, google, google académico, google imágenes, YouTube y paginas especializadas como

www.lasegundaguerra.com,www.segundaguerramundial.es,www.eurasia1945.com, www.ushmm.org, www.biografiasyvidas.com especializadas en el tema.

Para la elaboración del video producto final del guion de trabajo metodología por proyectos los estudiantes utilizan los programas predeterminados de Windows Movie Maker año 2010, 2016, Microsoft Power Point año 2016, 2017, viva video, el editor de video preestablecido en los celulares entre otros.

El análisis descriptivo de la información se realizó empleando el programa estadístico r 35, el cual permitió valorar medidas de tendencia central y grado de dispersión a través de los siguientes estadísticos primer cuartil, tercer cuartil, el promedio, la mediana, el índice t-Student, desviación estándar, los cuales permitieron analizar y comparar los resultados obtenidos por los dos grupos.

\section{Resultados}

Tabla 1 Comparación de los resultados grupo Control y Experimental 


\begin{tabular}{|c|c|c|c|c|c|c|c|c|c|c|}
\hline \multicolumn{10}{|c|}{ Resultados obtenidos grupo Control y Experimental } \\
\hline \multirow{2}{*}{ Grupo } & & Min. & $\begin{array}{c}1 \text { st } \\
\text { Qu. }\end{array}$ & Mediana & Media & $\begin{array}{c}\text { 3er } \\
\text { Qu. }\end{array}$ & Máx. & SD & Aprob. & Reprob. \\
\hline \multirow{3}{*}{ Control } & Pretest & 7 & 10 & 11 & 11 & 12 & 17 & 2.40 & 3 & 20 \\
\cline { 2 - 12 } & Postest & 9 & 12 & 13 & 13 & 15 & 18 & 2.56 & 8 & 15 \\
\cline { 2 - 12 } & Diferencia & 2 & 2 & 2 & 2 & 3 & 1 & 0.16 & 5 & 5 \\
\hline
\end{tabular}

\begin{tabular}{|c|c|c|c|c|c|c|c|c|c|c|}
\hline \multirow{3}{*}{ Experimental } & Pretest & 4 & 7 & 11 & 10 & 14 & 17 & 4.37 & 5 & 18 \\
\cline { 2 - 11 } & Postest & 7 & 11 & 14 & 13 & 15 & 17 & 2.77 & 9 & 14 \\
\cline { 2 - 11 } & Diferencia & 3 & 4 & 3 & 3 & 1 & 0 & 1.60 & 4 & 4 \\
\hline
\end{tabular}
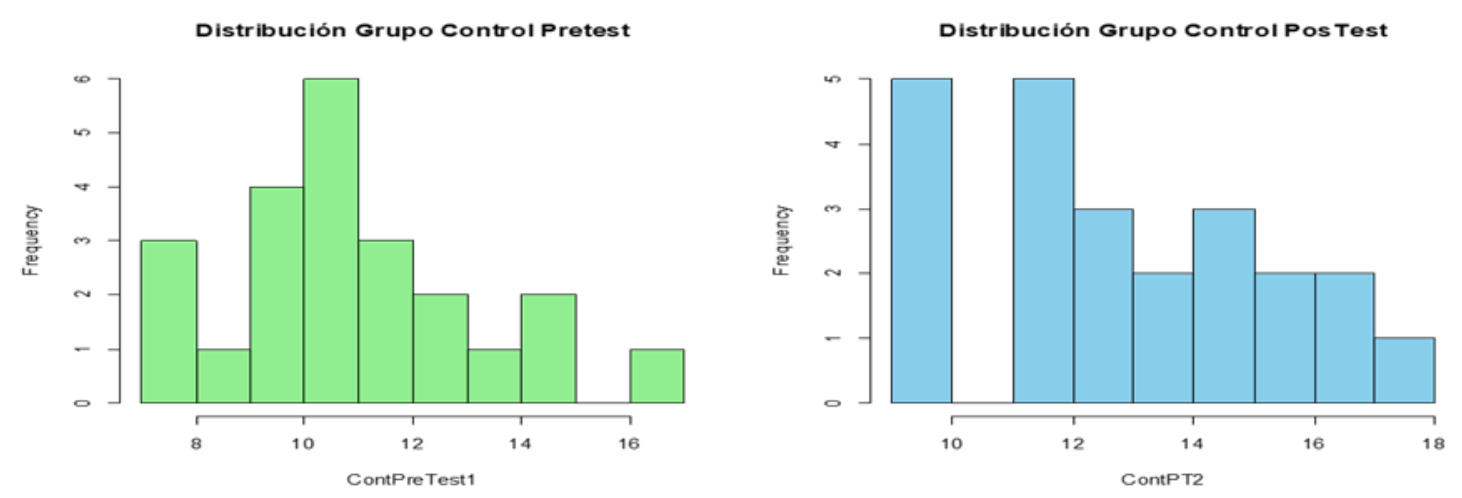

Gráfico 1 Frecuencia de distribución grupo control
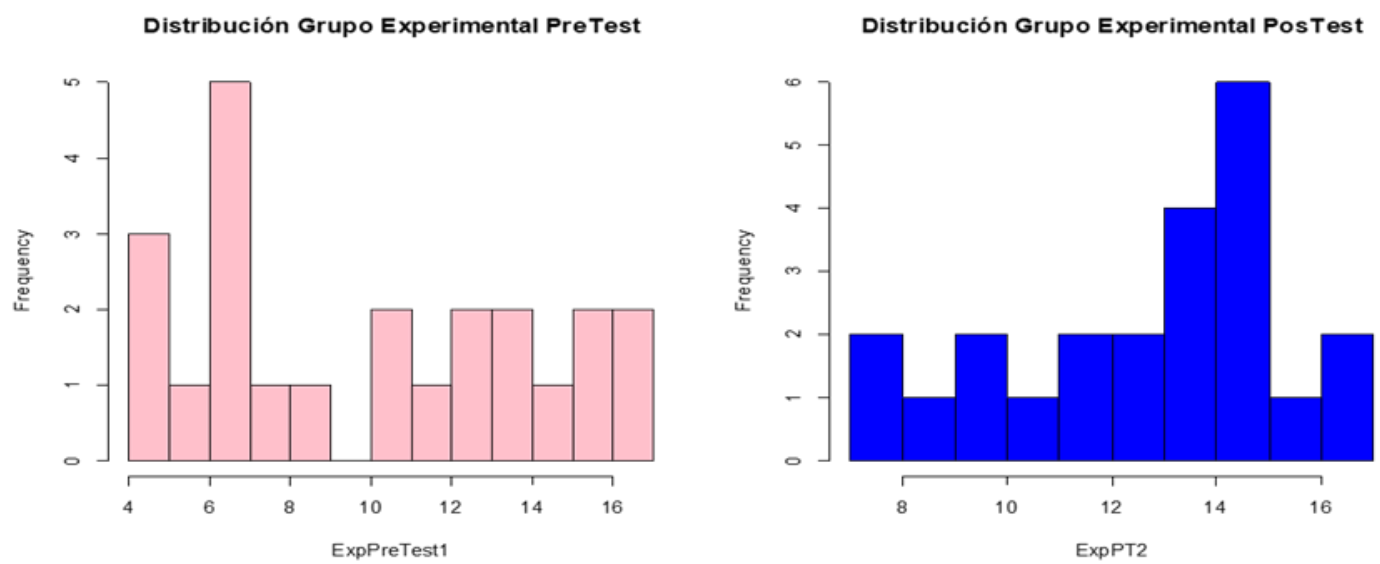

Gráfico 2 Frecuencia de distribución grupo experimental 


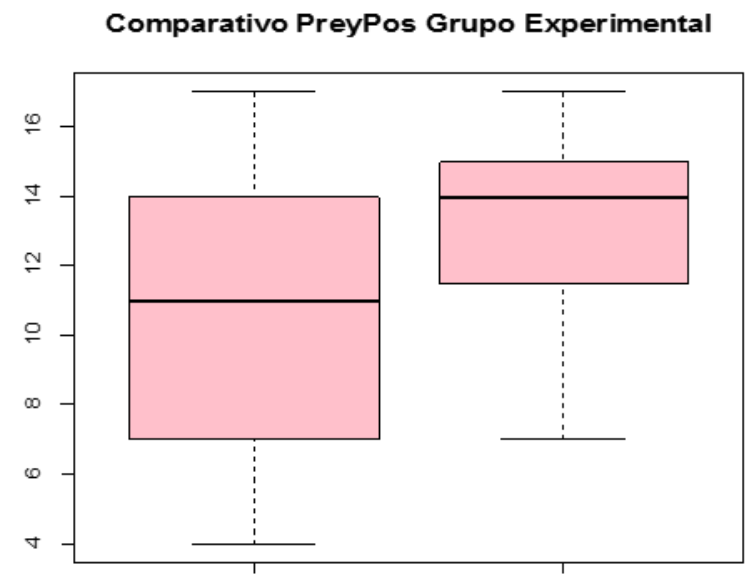

Gráfico 3 Diagrama de cajas comparativo pretest y postest grupo experimental

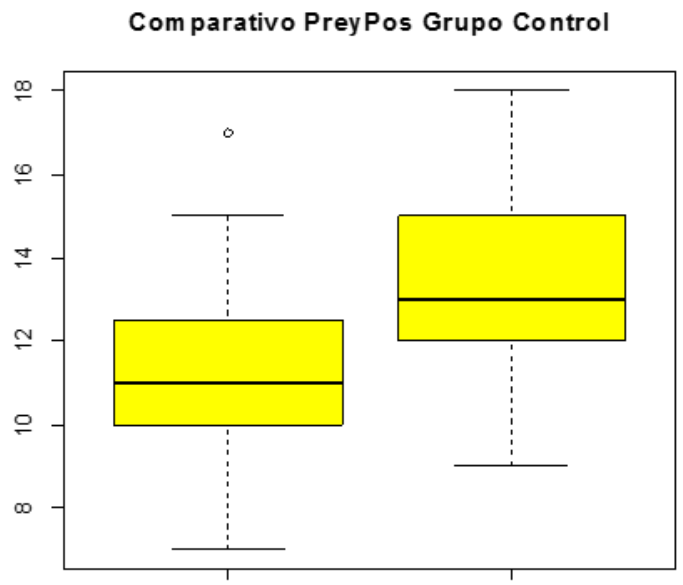

Gráfico 4 Diagrama de cajas comparativo pretest y postest grupo control

\section{Análisis de resultados}

Luego de emplear las estrategias didácticas y aplicar los instrumentos a los grupos control y experimental con el fin de determinar los conocimientos previos y adquiridos, se encontraron los siguientes resultados:

En el guion de trabajo metodología por proyectos los estudiantes una vez reciben las orientaciones inician su trabajo en los grupos de manera autónoma desarrollando así las fases establecidas. El trabajo fue realizó bajo la orientación y guía del docente, aunque se debe tener en cuenta que la mayoría se desarrolló de manera autónoma, de allí que la labor docente fue la de aclarar inquietudes relacionadas con el tema y uso del software, se resalta el trabajo entre estudiantes ya que estos se apoyaban con los demás grupos cuando tenían una dificultad o duda. Como fortaleza de la metodología se encuentra que motiva a los estudiantes para el desarrollo del trabajo, logro de las metas propuestas, permite el uso de los recursos propios y de la institución (Tablet, computadores, celulares). 
En los resultados del grupo control aprendizaje por el sistema tradicional tabla 1. En el primer cuartil se evidencia que 6 estudiantes obtuvieron resultados menores a 12 peguntas correctas lo cual comparado con el pretest se evidencia que mejoraron dos estudiantes. A diferencia del tercer cuartil en el que 17 estudiantes obtuvieron resultados inferiores a 15 preguntas correctas en promedio que comparado con el pretest el aumento fue de 3 estudiantes.

Analizando el grafico 1. Y la desviación estándar tabla 1, se evidencia que en el pretest fue de 2.4 y en el postest 2.56, siendo la diferencia 0.16 lo cual quiere decir que en el pretest el grupo a nivel general en cuanto a los resultados era más compacto a diferencia del postest en el que el grupo fue más disperso, lo que indica que los estudiantes con resultados buenos y bajos aumentaron en un rango mínimo, por tanto se abre una brecha de 0.16 en relación con el pretest, siendo así que luego de la enseñanza con el método tradicional el grupo fue más disperso respecto a sus puntajes de la prueba, ello lo podemos corroborar con el grafico 1.

De Acuerdo con la cantidad de preguntas los estudiantes que aprueban el examen en el pretest es de 3 estudiantes y en postest 8 estudiantes lo que indica que la mejoría fue de 5 estudiantes; en cambio, en la cantidad de estudiantes desaprobados hubo una reducción de 5 estudiantes ya que en la prueba inicial fueron 20 y en la prueba final 15 los que no aprobaron el examen. Por lo tanto, de acuerdo a la media, el grupo a nivel general tiene una mejoría en 2 pasando de 11 a 13 preguntas correctas en promedio en el postest.

En la grafico 3. Se evidencia a nivel general como mejoraron los resultados luego de la enseñanza con el método tradicional, lo anterior se corrobora en cada una de las variables tenidas en cuenta en la tabla 1. (mínimo, cuartil, mediana, media, máximo, desviación estándar), pero a su vez podemos notar con el crecimiento de la desviación estándar que las diferencias existentes entre los estudiantes que se pueden catalogar como buenos y regulares académicamente se hace más notoria, es decir los dos mejoran, pero, los buenos mejoran aún más académicamente.

En los resultados del grupo experimental aprendizaje mediante las estrategias didácticas de las TIC, metodología por proyectos, trabajo colaborativo-cooperativo, en la tabla 1. Primer cuartil podemos ver que 6 estudiantes obtienen resultados inferiores a 11 preguntas correctas que comparado con el pretest mejoran 4 estudiantes. En contrasté al tercer cuartil en el que 17 estudiantes obtienen resultados inferiores a 15 preguntas correctas lo cual quiere decir que hubo una mejoría de 1 en relación al pretest

En el grafico 2. se presenta la frecuencia de distribución del grupo experimental en y según la desviación estándar, en el pretest 4.31 y en el postest 2.77, podemos evidenciar una diferencia significativa respecto a los estudiantes con alto y bajo rendimiento; de acuerdo a los resultados del postest se da una mejoría en todo el grupo ya que según la desviación estándar del 2.77, el grupo después de emplear las TIC, metodología proyectos, trabajo colaborativo y cooperativo como estrategias didácticas para el aprendizaje del tema Segunda Guerra Mundial, se compacta, lo que indica que los estudiantes obtuvieron resultados similares, por tanto la brecha que se evidenció en el pretest, en el que la dispersión del grupo era alta (4.31) comparada con el postest se reduce 1.54, por eso luego de emplear el método de enseñanza alternativo, el grupo se compacta lo cual indica que los estudiantes obtuvieron resultados similares por tanto 
disminuye la cantidad de estudiantes con respuestas incorrectas y aumenta los de respuestas correctas de forma significativa.

Luego de hacer la revisión de los cuestionarios, el pretest lo aprueban 5 estudiantes y el postest 9 lo que indica que mejoraron 4 estudiantes; en contraste la cantidad de estudiantes que no aprobaron el examen en el pretest fue de 18 y en el postest 14, de ello podemos enuncia que mejoraron 4 estudiantes en relación a la cantidad que no aprobaron el examen en el pretest.

En la gráfica 4 la incidencia a nivel general fue positiva (mejoría) en los resultados que obtuvo el grupo experimental luego de aplicar las estrategias didácticas de la TIC, la metodología por proyectos, el trabajo colaborativo y cooperativo como estrategias didácticas para el aprendizaje del tema Segunda Guerra Mundial, ya que en cada una de las variables (mínimo, cuartil, mediana, media, máximo, desviación estándar) el cambio fue notable, según lo podemos ver en la gráfica. En el que el grupo de acuerdo al grafico después de emplear estas estrategias didácticas obtiene resultados similares.

\section{Discusión de Resultados}

En la investigación el aprendizaje de la historia con wiki en educación secundaria realizada por Mur, L (2015) se emplea un sistema colaborativo de aprendizaje mixto que combina el modelo presencial con el virtual el cual tiene como fin transformar el rol del profesor tradicional a uno e-tutor. Este trabajo es desarrollado con una muestra de setenta y un estudiantes pertenecientes al grado séptimo los cuales mediante el desarrollo de veintidós secciones de aprendizaje divididas en fases de trabajo (cuestionario inicial, diario del investigador, cuestionario final, cuestionario de elementos usados en la wiki), en las cuales se desarrolla el tema edad media, del cual como producto final mediante grupos colaborativos se diseña una wiki y luego se expone su contenido.

En la investigación la enseñanza de la historia a través de las tecnologías, la creatividad y el trabajo colaborativo realizada por Moreno, J.; Vera, M.; Soriano, M.; Seva, F.; Quiñonero, F.; Ibáñez, M. (2015) se establece como hipótesis que mediante el empleo de una metodología innovadora en el aprendizaje por descubrimiento se desarrolla el pensamiento crítico -reflexivo y se mejoran los resultados. Según el autor lo anterior tiene su fundamento en el desarrollo de diferentes estrategias metodológicas como el puzle, video on line, veo, elaboración de un tríptico o redacción de un cuento. Por ello mediante el empleo de programas se logra desarrollar el pensamiento crítico, reflexivo.

El punto común en las investigaciones realizadas se encuentra en el aumento de la motivación en los estudiantes debido al cambio metodológico, como queda evidenciado cuando se emplea la wiki, el trabajo colaborativo y se da el proceso de transición entre el método tradicional a uno que integra estrategias didácticas que responden a necesidades educativas actuales.

Es de anotar que las investigaciones parten del modelo de enseñanza tradicional en el que el profesor es el poseedor del conocimiento, la disciplina y el estudiante un agente pasivo atento a recibir y cumplir órdenes. Por ello según lo demostrado en las investigaciones el nuevo modelo 
de enseñanza exige cambiar la concepción que se tiene sobre la efectividad de las nuevas estrategias de enseñanza ya que según lo demostrado son efectivas en el ámbito académico y actitudinal.

\section{Conclusiones}

El método de aprendizaje alternativo las TIC, la metodología por proyectos, el trabajo colaborativo-cooperativo genera motivación, interés, deseo de participar y disposición en los estudiantes para asistir a clase y aprender debido a la metodología empleada y elementos tecnológicos utilizados (celular, Tablet, computador portátil) con los cuales se dinamiza y amplía la motivación por aprender sobre la temática, donde como efecto se evidencia una sobresaliente mejoría a nivel académico y disciplinario.

Por medio de la implementación de la metodología alternativa los estudiantes se motivan por trabajar en proyectos, ampliar los conocimientos en el tema; es importante resaltar que dentro de las fuentes utilizadas se encuentra: educatina, Wikipedia, slideshare, google, google imágenes, google académico, YouTube, páginas especializadas en la temática entre otros.

La implementación de las TIC, la metodología por proyectos, el trabajo colaborativocooperativo promueven la autonomía en los estudiantes a la hora de ejecutar la guía, evidenciado en el uso responsable del tiempo, por tanto mediante esta metodología no hubo momentos en los que los elementos distractores estuvieran por encima de lo programado (uso de celular, elementos distractores, juegos), además, cuando surgían inquietudes en su mayoría eran resueltas con los demás grupos y de forma esporádica con el docente.

La principal dificultad estuvo relacionada con la capacidad del internet, ya que en varias ocasiones la red en lapsos cortos se saturaba, por ello como plan de contingencia ante esta dificultad temporal, se solicitó a los estudiantes traer información (texto, videos, mapas, podcast, capturas a la pantalla) desde las casas almacenada en los dispositivos móviles USB, celulares, Tablet, portátil o impresa. La cual se utilizaba en la clase para el desarrollo pleno de la guía. Se resalta la creatividad y empeño de los estudiantes por el desarrollo del trabajo el cual queda evidenciado por los detalles estéticos del video realizado por los estudiantes del grupo experimental sobre la Segunda Guerra Mundial.

Actualmente enseñar/desarrollar competencias en ciencias sociales y lograr un aprendizaje significativo en los estudiantes puede llegar a convertirse en un reto, esta afirmación tiene su fundamento, porque una parte de jóvenes de hoy tienen cierta apatía por lo académico. Es por ello que una actividad que implique procesos de pensamiento crítico hace que el joven se vea obligado a desistir de las actividades asignadas por el docente. Por tanto, en casos como este, se debe hacer que el joven se enamore del área y para ello es importante que cambie su concepción respecto a la asignatura, que para el caso mediante el empleo de un método de enseñanza distinto al que estaba acostumbrado el estudiante (método tradicional), por uno que integró estrategias didácticas distintas, como fue el caso del grupo experimental con el cual se utilizaron las TIC, la metodología por proyectos y el trabajo colaborativo - cooperativo para realizar el proceso de aprendizaje del tema Segunda Guerra Mundial en el área de ciencias sociales, los resultados van a ser mejores como a los del grupo control. 


\section{Bibliografía}

Austin, V., Abella, V., Delgado, V., \& Hortiguela, D. (2016). Aprendizaje basado en proyectos a través de las TICS. Una experiencia de innovación docente desde las aulas universitarias. Formación Universitaria, 31 - 38.

Cabero Almenara, J. (2014). Nuevas miradas sobre las TIC en la educación. Revista Andalucía Educativa.

Casadiego Cabrales, A. M., \& Vera Silva, A. A. (2016). Efectividad del uso de herramientas de la web utilizando la estrategia de aprendizaje colaborativo. En N. E. López Jiménez, \& A. Pérez 87

Castro, La evaluación y las TIC: procesos determinantes de la realidad educativa actual (pág. 295). Neiva: Centro de Investigaciones en Calidad de la Educación.

CIDEAD. (2005). La Segunda Guerra Mundial y sus Consecuencias. España: CIDEAD.

Fernández Muñoz, R. (2010). Competencias Profesionales del Docente en el siglo XXI. Aplicadas a la Educación.

Landow, G. (1995). Hipertexto. España: Ediciones Paidós.

Maldonado Pérez, M. (2008). Aprendizaje basado en proyectos colaborativos, una experiencia en educación superior. Revista de Educación Laurus, 158 - 180.

Marín Gallego, J. D. (2012). La investigación en educación y pedagogía. Bogotá: USTA.

Martínez Hernández, B. L., \& Gómez Díaz, M. (2014). Las TIC como herramienta para fortalecer el aprendizaje colaborativo, en las IES. Centro de Estudios e Investigaciones para el Desarrollo Docente CENID, 11.

Marc Prenskey, (2010). Teaching Digital Natives: Partnering for real learning. London: Corwin

Monsalve Ochoa, M. L. (2011). Implementación de las TIC como estrategia didáctica para generar un aprendizaje significativo.

Morrissey, J. (2010). El uso de TIC en la enseñanza y el aprendizaje. Cuestiones y desafíos. 235 - 246.

Mur Sangra, L. (2015). El aprendizaje de la historia con wiki en educación secundaria. Investigación Didáctica, 9.

Murcia Castellanos, Y. C., Tejedor Estupiñan, M. L., \& Lancheros Cuesta, D. Y. (2017). Impacto de una herramienta multimedial en el proceso de enseñanza-aprendizaje de la historia en el aula. Revista de Medios y Educación, 201-228.

Villar Solo, S. (2013). Aprendizaje basado en proyectos. 19 pág. 